GEOMETRY IN NONLINEAR CONTROL

AND DIFFERENTIAL INCLUSIONS

BANACH CENTER PUBLICATIONS, VOLUME 32

INSTITUTE OF MATHEMATICS

POLISH ACADEMY OF SCIENCES

WARSZAWA 1995

\title{
CONTROL STRUCTURES
}

\author{
ROBERT L. BRYANT \\ Department of Mathematics, Duke University \\ P.O. Box 90320, Durham, North Carolina 27r08-0320, U.S.A. \\ E-mail: bryant@math.duke.edu \\ ROBERT B. GARDNER \\ Department of Mathematics, University of North Carolina \\ Chapel Hill, North Carolina 27599-3250, U.S.A. \\ E-mail: rbg@math.unc.edu
}

\begin{abstract}
We define an extension of the classical notion of a control system which we call a control structure. This is a geometric structure which can be defined on manifolds whose underlying topology is more complicated than that of a domain in $\mathbb{R}^{n}$. Every control structure turns out to be locally representable as a classical control system, but our extension has the advantage that it has various naturality properties which the (classical) coordinate formulation does not, including the existence of so-called universal objects and classifying maps. This more general viewpoint simplifies the study of the invariants of even classical control systems. Its main technical advantage is that tools like the method of equivalence can be directly and easily applied to the study of control structures.
\end{abstract}

\section{Introduction}

Classical control systems. The problem of how to give a useful, intrinsic, and coordinate-free description of control systems and feedback equivalence has been considered by Brockett [2], but his formulation covers only the control linear systems. Our aim in this section is to give a formulation which extends to the general non-linear control system.

We recall that a classical control system on a domain in $n$-space is simply an

1991 Mathematics Subject Classification: 93B27.

Partially supported by NSF grant DMS-9205222.

Partially supported by NSF grant DMS-9204942.

The paper is in final form and no version of it will be published elsewhere. 
underdetermined system of ordinary differential equations

$$
\frac{d x}{d t}=F(x, u), \quad x \in \mathbb{R}^{n}, u \in \mathbb{R}^{m},
$$

where $x=\left(x^{i}\right)$ represents the state space variables and $u=\left(u^{a}\right)$ represents the control variables. To avoid trivial degenerations, we assume that, for each $x_{0} \in \mathbb{R}^{n}$, the map $u \mapsto F\left(x_{0}, u\right)$ is an embedding of $\mathbb{R}^{m}$ into $\mathbb{R}^{n}$ (i.e., the controls should appear non-degenerately in the equations). Such a system can be thought of as defining an $(n+m)$-dimensional submanifold $M$ of the tangent bundle $T \mathbb{R}^{n} \simeq$ $\mathbb{R}^{n} \times \mathbb{R}^{n}$ of the state space $\mathbb{R}^{n}$. Namely, $M$ is the image of the mapping

$$
V: \mathbb{R}^{n} \times \mathbb{R}^{m} \longrightarrow T \mathbb{R}^{n}
$$

defined by

$$
V(x, u)=\sum_{i=1}^{n} F^{i}(x, u) \frac{\partial}{\partial x^{i}} .
$$

Our assumption of no trivial degenerations implies that $M$ is a submanifold of $T \mathbb{R}^{n}$ and $V$ is a regular parametrization of it.

An integral curve (or "control curve") of the system (1) is a curve

$$
\gamma: \mathbb{R} \longrightarrow \mathbb{R}^{n},
$$

with the property that there exists another curve $\beta: \mathbb{R} \rightarrow \mathbb{R}^{m}$ so that

$$
\dot{\gamma}(t)=V(\gamma(t), \beta(t)),
$$

or equivalently $\gamma^{*} d x=F \circ(\gamma, \beta) d t$. Note that a curve $\gamma: \mathbb{R} \rightarrow \mathbb{R}^{n}$ is a control curve if and only if its tangential lifting $\dot{\gamma}: \mathbb{R} \rightarrow T \mathbb{R}^{n}$ has its image in $M$.

In the study of systems such as (1), a common first step is to try to change coordinates so as to place the system into some more tractable "normal form", indeed, to linearize it if possible. The sort of changes of coordinates one allows for reaching this normal form depends on the nature of the specific problem, but frequently one allows changes of coordinates of the form

$$
(X, U)=\Phi(x, u)=(\phi(x), \psi(x, u)),
$$

i.e., the so-called "feedback" transformations. Transformations of the form (2) which preserve the system (1) are called the feedback symmetries of the system. Geometrically, these are the changes of coordinates which carry integral curves of the system to integral curves of the system.

These transformations are particularly of interest in the case that the control system is generated from a so-called "constraint" problem, i.e., one in which the control curves are defined by restrictions on their possible tangent vectors. In this case, the specific way in which the constraints are coordinatized is not a geometrically meaningful part of the problem and so feedback transformations are a natural class to consider.

Control systems on manifolds. Now, in many interesting control theory problems, the natural state space has topological features which prevent it from being 
identified with a domain in some $\mathbb{R}^{n}$. For example, in the classical problem of understanding a ball rolling on a plate without twisting or slipping, the natural state space is $\mathrm{SO}(3) \times \mathbb{R}^{2}$, the first factor $\mathrm{SO}(3)$ representing the orientation of the ball and the second factor $\mathbb{R}^{2}$ representing the point of contact of the ball with the plate. Also, in many problems involving autopilots for helicopters or other flying objects, the natural state space contains an $\mathrm{SO}(3)$-factor which represents the orientation of the object. Such examples point out the need to have a control theory for which the state space is allowed to be a more general manifold than just a domain in some $\mathbb{R}^{n}$.

A natural generalization of the classical control system to the case of control systems defined on manifolds is the following one. Fix a manifold $X$ and consider a subset $M \subset T X$, where $T X$ is the tangent bundle of the manifold $X$. We say that a curve

$$
\gamma: \mathbb{R} \longrightarrow X
$$

is an $M$-curve if its tangential lift $\dot{\gamma}: \mathbb{R} \rightarrow T X$ has its image in $M\left({ }^{1}\right)$. Extending the classical terminology, we will refer to $X$ as the state space and to $M$ as the configuration space.

For example, in the case of a ball rolling on a plate without slipping or twisting, a case considered recently by Brockett and Dai [2a], the state space is $X=$ $\mathrm{SO}(3) \times \mathbb{R}^{2}$ and the configuration space $M \subset T X$ turns out to be a (linear) subbundle of $T X$ of rank 2 which is transverse to the $\mathrm{SO}(3)$-leaves. In local coordinates, of course, this fact will be expressed by saying that the system is control linear.

Generalizing the notion of feedback equivalence, we say that two such structures $\left(X_{1}, M_{1}\right)$ and $\left(X_{2}, M_{2}\right)$ are equivalent if there exists a diffeomorphism $f: X_{1} \rightarrow X_{2}$ with the property that $f_{*}\left(M_{1}\right)=M_{2}$. Clearly any such equivalence between two such structures carries $M_{1}$-curves in $X_{1}$ to $M_{2}$-curves in $X_{2}$. In fact, under mild regularity assumptions on $M_{1}$ and $M_{2}$ (for example, the assumptions that each of the $M_{i}$ be a submanifold and that each of the basepoint mappings $M_{i} \rightarrow X_{i}$ be a surjective submersion are sufficient), any diffeomorphism $f: X_{1} \rightarrow X_{2}$ which identifies the class of $M_{1}$-curves with the class of $M_{2}$-curves must necessarily satisfy $f_{*}\left(M_{1}\right)=M_{2}$ and hence be an equivalence between the two structures in this sense.

The geometry of the tangent bundle. It is natural to try to derive necessary and sufficient conditions for equivalence between two structures $\left(X_{1}, M_{1}\right)$

$\left({ }^{1}\right)$ Caveat: To avoid confusing our readers, we want to explicitly point out that the tangential lift $\dot{\gamma}$ is not just a map to a fixed vector space, but includes the base point information as well. E.g., in the most elementary case, where $X=\mathbb{R}^{n}$, we have $T X=\mathbb{R}^{n} \times \mathbb{R}^{n}$ and the tangential lift of $\gamma: \mathbb{R} \rightarrow \mathbb{R}^{n}$ is the curve $\dot{\gamma}: \mathbb{R} \rightarrow \mathbb{R}^{n} \times \mathbb{R}^{n}$ given by the formula

$$
\dot{\gamma}(t)=\left(\gamma(t), \gamma^{\prime}(t)\right) \text {. }
$$

Of course, the classical notation for curves in $n$-space does not have to preserve any distinction between $\dot{\gamma}$ and $\gamma^{\prime}$, but for curves in more general manifolds, this distinction is very important. 
and $\left(X_{2}, M_{2}\right)$. Our point of view in this paper will be to describe a natural geometric structure induced on submanifolds $M \subset T X$ which encodes the complete geometry of the pair $(X, M)$. This geometric structure is what we shall call a control structure.

Before we can understand what sort of structure a submanifold $M$ of a tangent bundle $T X$ might inherit, it will be helpful to review the geometry of tangent bundles. Specifically, by "geometry" in this case, we mean the geometric features of the tangent bundle $T X$ which are preserved by the tangential diffeomorphisms of $T X$ i.e, the ones which are induced by the diffeomorphisms of the base manifold $X$. The geometry (in this sense) of the cotangent bundle is familiar, its study leading up to the edifice of symplectic geometry. By contrast, the geometry of tangent bundles, while just as fundamental, is not as well-known.

Before discussing this geometry, we want to introduce the concept of natural coordinates on tangent bundles. If $V \subset X$ is an open set on which there exists a coordinate chart $x: V \rightarrow \mathbb{R}^{n}$, then there is a natural (tautological) extension of these coordinates to a coordinate chart

$$
(x, u): T V \rightarrow \mathbb{R}^{n} \times \mathbb{R}^{n}
$$

on the tangent bundle of $V$. This extension is uniquely characterized by the condition that, for any curve $\gamma: \mathbb{R} \rightarrow V$, the curve $\dot{\gamma}: \mathbb{R} \rightarrow T V$ satisfies

$$
((x, u) \circ \dot{\gamma})(t)=\left((x \circ \gamma)(t),(x \circ \gamma)^{\prime}(t)\right) .
$$

We shall use these coordinates from time to time.

Now, one obvious feature of $T X$ which is preserved is the vector bundle structure, i.e, the fibers of the base-point mapping $T X \rightarrow X$ and the linear structure on those fibers. This linear structure will, of course, not be inherited by submanifolds $M \subset T X$, but, in the case that $M$ is a submanifold and the induced basepoint mapping $M \rightarrow X$ is a surjective submersion, $M$ will be foliated by its intersections with the fibers of the mapping $T X \rightarrow X$ and the leaves of this foliation will have codimension $n$.

Another obvious geometric feature of $T X$ is its zero section, which is clearly preserved under diffeomorphisms induced from $X$. For this reason, the geometry of submanifolds passing through the zero section is different from the geometry of submanifolds of the punctured tangent bundle $T^{\circ} X$, i.e., $T X$ minus its zero section. This corresponds to the fact that the geometry of control systems such as (1) is quite different at so-called "rest points", i.e., points in $x u$-space where the function $F$ vanishes. Throughout this paper, we are going to concentrate on the geometry away from the rest points, so, properly speaking, we need to understand the geometry of the punctured tangent bundle.

Let $I$ denote the module of 1 -forms $\omega$ on $T^{\circ} X$ which have the property that for any immersed curve $\gamma: \mathbb{R} \rightarrow X$, the curve $\dot{\gamma}: \mathbb{R} \rightarrow T^{\circ} X$ satisfies $\dot{\gamma}^{*}(\omega)=0$. In natural coordinates, it is easy to see that $I$ is generated by the 1 -forms

$$
u^{i} d x^{j}-u^{j} d x^{i}
$$


In fact, on the open set where, say $u^{1} \neq 0$, the module $I$ is generated by the $n-1$ forms

$$
\theta^{i}=d x^{i}-\left(u^{i} / u^{1}\right) d x^{1}, \quad \text { for } i=2,3, \ldots, n .
$$

By its very construction, $I$ does not depend on any choice of coordinates and hence must be preserved under all tangential diffeomorphisms.

Also, let $A$ denote the set of 1 -forms $\phi$ on $T^{\circ} X$ which have the property that for any immersed curve $\gamma: \mathbb{R} \rightarrow X$, the curve $\dot{\gamma}: \mathbb{R} \rightarrow T^{\circ} X$ satisfies $\dot{\gamma}^{*}(\phi)=d t$.

Clearly, the difference $\phi_{1}-\phi_{2}$ of any two elements of $A$ is an element of $I$ and conversely, if $\phi$ is in $A$ and $\omega$ is in $I$ then $\phi+\omega$ lies in $A$.

What is not clear is that $A$ is non-empty. However, in a natural coordinate chart, it is easy to write down an example, just take

$$
\phi_{0}=\frac{u^{1} d x^{1}+\cdots+u^{n} d x^{n}}{\left(u^{1}\right)^{2}+\cdots+\left(u^{n}\right)^{2}} .
$$

From this, by a partition of unity argument, one could define such an $\phi$ on the entire $T^{\circ} X$. Alternatively, if one fixes a Riemannian metric $g$ on $X$ whose expression in a local coordinate chart takes the form

$$
g=g_{i j}(x) d x^{i} d x^{j}
$$

then one can write down the local expression

$$
\phi_{0}=\frac{g_{i j}(x) u^{i} d x^{j}}{g_{i j}(x) u^{i} u^{j}}
$$

and verify that $\phi_{0}$ is well-defined independent of the choice of local coordinates $x$ and lies in $A$.

Since $A$ is non-empty, we can symbolically write $A=\phi+I$ where $\phi$ is any element of $A$. It is also customary to write $A=[\phi]$ for some (and hence any) element $\phi \in A$, where the square brackets denote the equivalence class of $\phi$ modulo $I$. Note that $\phi$ does not extend smoothly (or even continuously) across the zero section of $T X$. Since $A$ is defined without reference to any choice of coordinates, it follows that $A$ must also be invariant under any any tangential diffeomorphism. Thus $A$ is also part of the geometry of the punctured tangent bundle.

One important property of the pair $(I,[\phi])$ should be noted: If we set

$$
J=C^{\infty}\left(T^{\circ} X\right) \cdot \phi+I,
$$

then $J$ is simply the completely integrable Pfaffian system of rank $n$ whose leaves are the fibers of the base point submersion $T^{\circ} X \rightarrow X$. (The reader can easily check this in the local coordinates given above. Also, see the next section.)

Control structures. We are interested in the geometry of submanifolds of $T^{\circ} X$. The advantage of working with differential forms and Pfaffian systems (as opposed to, say, vector fields and distributions) is that they pull back naturally to submanifolds. The submanifolds of interest in this case are the $(n+m)$-dimensional 
submanifolds $M \subset T^{\circ} X$ with the property that the base point mapping $M \rightarrow X$ is a surjective submersion. Such submanifolds, which we shall call allowable, inherit the Pfaffian system $I$ and its "affine translate" $A=[\phi]$ directly by pull-back. We are going to see that this inherited geometry suffices to determine $M$ uniquely up to feedback equivalence.

Our discussion so far motivates the following definition:

Definition. A control structure (of rank $n$ ) on a manifold $M$ of dimension $n+m$ is a pair $(I,[\phi])$, where $I$ is a Pfaffian system of rank $n-1$ and $\phi$ is a 1-form on $M$ well-defined up to addition of an element of $I$, with the property that the Pfaffian system $J$ spanned by $I$ and $\phi$ is everywhere of rank $n$ and is completely integrable. The leaf space of $J$, denoted by $X$, is called the state space. We say that the control structure is amenable if $X$ is a smooth manifold with its natural topology and smooth structure.

Note that, by its very definition, the punctured tangent bundle of any smooth manifold has a canonical control structure, which we sometimes refer to as the "tautological" or "universal" control structure.

The strength of the general concept will be seen when we establish the following result:

Given an amenable control structure $(I,[\phi])$ on a smooth manifold $M$, there is a canonical smooth map

$$
\widehat{x}: M \longrightarrow T^{\circ} X
$$

which pulls back the universal control structure on $X$ to the given control structure on $M$.

(Note that for any control structure as we have defined it, the underlying manifold $M$ will be covered by open sets to which the control structure restricts to become amenable.)

Integral curves and equivalences. The notion of integral curve naturally generalizes to control structures.

Definition. An integral curve of a control structure $(I,[\phi])$ on a manifold $M$ is a differentiable curve $\gamma: \mathbb{R} \rightarrow M$ satisfying

$$
\gamma^{*} I=0 \text { and } \gamma^{*} \phi=d t .
$$

Moreover, the transformations which identify the integral curves of one control structure with those of another are easily characterized:

Definition. A feedback equivalence between control structures $\left(M_{1}, I_{1},\left[\phi_{1}\right]\right)$ and $\left(M_{2}, I_{2},\left[\phi_{2}\right]\right)$ is a diffeomorphism $f: M_{1} \rightarrow M_{2}$ which satisfies $f^{*}\left(I_{2}\right)=I_{1}$ and

$$
f^{*}\left(\phi_{2}\right) \equiv \phi_{1} \text { modulo } I_{1}
$$

We then have the following theorem, which, in the case of classical control systems, is found in Gardner and Shadwick [4]. 
TheOREM. If $M_{1}$ and $M_{2}$ are $(n+m)$-manifolds endowed respectively with control structures $\left(I_{1},\left[\phi_{1}\right]\right)$ and $\left(I_{2},\left[\phi_{2}\right]\right)$ respectively, then a diffeomorphism $f$ : $M_{1} \rightarrow M_{2}$ is a feedback equivalence if and only if $f$ identifies the integral curves of the two structures.

Proof. By standard ODE arguments, it is easy to see that for any control structure $(I,[\phi])$ on a manifold $M$ and any vector $v \in I^{\perp} \subset T M$, there exists an integral curve of $(I,[\phi])$ tangent to $v$.

Suppose first that $f$ identifies the integral curves of the two structures. Then, by the above argument, it must also identify $I_{1}^{\perp}$ with $I_{2}^{\perp}$ and hence $I_{1}$ with $I_{2}$. Now selecting an $\phi_{2}$ which represents $\left[\phi_{2}\right]$, we know that, for any integral curve $\gamma$ of $\left(I_{1},\left[\phi_{1}\right]\right)$ the map $f \circ \gamma$ must be an integral curve of $\left(I_{2},\left[\phi_{2}\right]\right)$. Thus,

$$
\gamma^{*}\left(f^{*}\left(\phi_{2}\right)-\phi_{1}\right)=(f \circ \gamma)^{*}\left(\phi_{2}\right)-d t=d t-d t=0 .
$$

Thus, it follows that

$$
\left[f^{*}\left(\phi_{2}\right)\right]=\left[\phi_{1}\right]
$$

Hence $f$ is a feedback transformation, as we wanted to show. The converse is, of course, trivial.

As will be seen, this result allows us to apply the method of equivalence directly to the problem of characterizing the invariants of control systems up to feedback equivalence. The characterization of feedback transformations by the method of equivalence [4], leads to the study of basic feedback invariant geometric objects associated to (1) and, more generally, to any control structure. Although in principle these structures could have been uncovered directly in an ad hoc way, it is important to know that a systematic tool was used to find them.

2. Explicit formulae for classical control systems. Before going on to the theoretical part of the discussion of control structures, we want to see what the objects $I$ and $[\phi]$ look like for a classical control system such as (1).

Consider the Pfaffian system $I$ defined as the set of 1 -forms on $\mathbb{R}^{n} \times \mathbb{R}^{m}$ which are linear combinations of the $d x$ and vanish on the integral curves of (1). This system has the simple direct description

$$
I=\left\{G d x \mid G: \mathbb{R}^{n} \times \mathbb{R}^{m} \rightarrow \mathbb{R}^{n} \text { such that } G F=0\right\} .
$$

This is easy to see since the condition $G F=0$ is precisely the condition needed in order that the condition $\gamma^{*} d x=(F \circ \gamma) d t$ should imply

$$
\gamma^{*}(G d x)=(G \circ \gamma) \gamma^{*} d x=(G \circ \gamma)(F \circ \gamma) d t=(G F) \circ \gamma d t=0 .
$$

This description also makes it clear that the system $I$ has rank $(n-1)$.

The second geometric object is the affine Pfaffian system $A$ of 1 -forms which are linear combinations of the $d x$ having the property that they pull back to become $d t$ along any integral curve of $I$. As noted above, since the difference of two elements of this affine system vanishes along any integral curve of $I$, the affine system $A$ can be written in the form $[\phi]=\phi+I$. 
An explicit representative for $\phi$ is given by

$$
\phi=\frac{F \cdot d x}{F \cdot F}
$$

where $\cdot$ is the usual euclidean dot product.

In particular, the system $J$ defined by

$$
J=I+\{\phi\}
$$

is the Pfaffian system of rank $n$ consisting of the linear combinations of $d x$. Thus, it is completely integrable and its leaf space is simply the space of state variables.

\section{Properties of control structures}

Universality. We can now prove the fundamental imbedding theorem for control structures.

Theorem. Let $(M, I,[\phi])$ be an amenable control structure with state space $X$. There is a canonical smooth mapping $\iota: M \rightarrow T^{\circ} X$ which pulls back the universal control structure on $T^{\circ} X$ to the given structure.

Proof. Let $\pi: M \rightarrow X$ be the submersion onto the leaf space. Now define $\iota: M \rightarrow T X$ by the rule

$$
\iota(m)=\pi_{*}\left\{w \in I_{m}^{\perp}(M) \mid \phi(w)=1\right\} .
$$

Let us first see why this is well defined. Now,

$$
\{\phi, I\}^{\perp}=\{d x\}^{\perp}=\operatorname{ker} \pi_{*} .
$$

It follows that, for any $w_{1}$ and $w_{2}$ in $I_{m}^{\perp}$ which satisfy and $\phi\left(w_{1}\right)=\phi\left(w_{2}\right)=1$, we must have $\pi_{*}\left(w_{1}-w_{2}\right)=0$. Thus,

$$
\iota(m)=\pi_{*}\left(w_{1}\right)=\pi_{*}\left(w_{2}\right) .
$$

Thus, $\iota$ is well defined.

It remains to be shown that the image of $\iota$ lies in $T^{\circ} X$. We will do this (and carry out the remainder of the proof) by introducing local coordinates. Fix a point $m_{0} \in M$ and let $x=\left(x^{1}, \ldots, x^{n}\right)$ be local coordinates on $X$ centered on $\pi\left(m_{0}\right)$. For notational simplicity, we will denote the functions $\pi^{*}\left(x^{i}\right)$ defined on a neighborhood of $m_{0} \in M$ simply by $x^{i}$. We now extend these $n$ functions (which vanish at $\left.m_{0}\right)$ to a local coordinate system $(x, u)=\left(x^{1}, \ldots, x^{n}, u^{1}, \ldots, u^{m}\right)$ centered at $m_{0}$. We now restrict our attention to the domain of this coordinate system.

Now, by construction, $I \subset \operatorname{span}\left\{d x^{1}, \ldots, d x^{n}\right\}$ and has rank $n-1$. By permuting the coordinates if necessary, we may assume that $\left(d x^{1}\right)_{m_{0}} \notin I_{m_{0}}$. It then follows that there are functions $F^{i}(x, u)$ for $i=2, \ldots, n$, so that

$$
I=\operatorname{span}\left\{d x^{2}-F^{2}(x, u) d x^{1}, \ldots, d x^{n}-F^{n}(x, u) d x^{1}\right\} .
$$


Moreover, since $I+[\phi]=\operatorname{span}\left\{d x^{1}, \ldots, d x^{n}\right\}$ it follows that there is a unique representative in $[\phi]$ of the form

$$
\phi=L(x, u) d x^{1}
$$

where $L$ is a non-vanishing function. It then follows that the vector field

$$
W=\frac{1}{L}\left(\frac{\partial}{\partial x^{1}}+F^{2} \frac{\partial}{\partial x^{2}}+\cdots+F^{n} \frac{\partial}{\partial x^{n}}\right)
$$

has the property that every element of $I$ vanishes on $W$ while $\phi(W)=1$. Thus, by our very definition of the map $\iota$, we have

$$
\iota(m)=W(m) \in T_{x(m)}^{\circ} X .
$$

Note that this shows that $\iota$ has image in $T^{\circ} X$, as we wished to show.

Now let $v^{1}, \ldots, v^{n}$ complete the $x^{i}$ to tangential coordinates on $T X$ as described above. Then, in these coordinates, $\iota$ has the formula

$$
(x(\iota(m)), v(\iota(m)))=\left(x^{i}(m), \frac{1}{L}, \frac{F^{2}}{L}, \ldots, \frac{F^{n}}{L}\right) .
$$

Now, in a neighborhood of $\iota\left(m_{0}\right)$, the universal control system $(\tilde{I},[\tilde{\phi}])$ on $T^{\circ} X$ is generated by

$$
\tilde{I}=\left\{d x^{2}-\left(v^{2} / v^{1}\right) d x^{1}, \ldots, d x^{n}-\left(v^{n} / v^{1}\right) d x^{1}\right\}
$$

and

$$
\widetilde{\phi}=\left(1 / v^{1}\right) d x^{1} .
$$

It is immediate from the definitions now that $\iota$ satisfies

$$
\iota^{*}\left(d x^{i}-\left(v^{i} / v^{1}\right) d x^{1}\right)=d x^{i}-F^{i} d x^{1}
$$

as well as $\iota^{*}(\widetilde{\phi})=L d x^{1}$. Thus, $\iota$ pulls the universal control structure on $T^{\circ} X$ back to the given one, as desired.

Non-degeneracy. Now, in fact, our definition of a control structure allows some undesirable behavior. For example, we could replace $M$ by $M \times P$ where $P$ is some manifold and let a new $I$ and $[\phi]$ just be the ones got by pulling back the ones on $M$ under the projection $M \times P \rightarrow M$. This would introduce $P$ as "spurious" controls, in much the same way as allowing a variable $u^{i}$ in the system (1) to not appear in the formulae for $F$. We want to avoid this sort of degeneracy, so we make the following definition.

Definition. We say that a control structure $(I,[\phi])$ on a manifold $M$ is nondegenerate if it cannot be written as the pull back of a control structure on $\bar{M}$ for some submersion $M \rightarrow \bar{M}$.

It is now an elementary result (which can be read off of the constructions in the proof just given) that the condition of being non-degenerate is equivalent to the (possibly locally defined) state space mapping $\iota$ being an immersion. Details will be left to the reader. 
Local realizability. We are now ready to discuss the relationship between control structures and control systems. The main result is the following one.

THEOREM. A non-degenerate control structure is locally equivalent to a classical control system of the form (1).

Proof. Fix a point in $M$ and let $U$ be a neighborhood on which the state foliation is amenable. Let $x$ be a vector of base coordinates on the leaf space $X$ and extend them by a vector of fiber coordinates $u$ to a coordinate system on $U$ (we may have to shrink $U$ to do this). If

$$
I=\left\{\omega^{1}, \ldots, \omega^{n-1}\right\} \quad \text { and } \quad J=\{d x\}
$$

on $U$, then we can write

$$
d x=F \phi+\sum_{j=1}^{n-1} G_{j} \omega^{j},
$$

where the independence of the $d x$ implies

$$
\operatorname{det}\left(F, G_{1}, \ldots, G_{n-1}\right) \neq 0 .
$$

Next, given a local integral curve $\gamma$ of the control structure, so that

$$
\gamma^{*} I=0 \text { and } \gamma^{*} \phi=d t,
$$

then

$$
\gamma^{*} d x=\gamma^{*}\left(F \phi+\sum_{j=1}^{n-1} G_{j} \omega^{j}\right)=(F \circ \gamma) d t,
$$

which verifies that $\gamma$ is an integral curve of the system (1).

Conversely given an integral curve of (1), it satisfies

$$
0=\gamma^{*} d x=\gamma^{*}\left(F(\phi)+\sum_{j=1}^{n-1} G_{j} \omega^{j}\right),
$$

and, by linear independence, this implies

$$
\gamma^{*} I=0 \quad \text { and } \quad \gamma^{*} \phi=d t
$$

as required.

The assumption of non-degeneracy now implies that the map given by $F$ induces a local immersion of $U$ into $T^{\circ} X$.

\section{The geometry of control structures}

A G-structure. As remarked in the introduction, this formalism places the problem of finding invariants under feedback transformation into the setting of control structures. We see immediately that defining a control structure on a manifold $M$ defines a $G$-structure with certain torsion (i.e., integrability) conditions. Now the method of equivalence can be applied to recover the results already achieved in special cases. The reader may consult the references for explicit results. 
Results. In the case of $n$ states and $(n-1)$ controls, this leads to the study of the fiber geometry of maps

$$
\mathbb{R}^{n} \times \mathbb{R}^{n-1} \rightarrow T^{\circ} \mathbb{R}^{n}
$$

which becomes the study of hypersurfaces in centro-affine geometry.

The first steps of the classification separate out the following cases:

I) Systems tangent to the radial direction, these are the non-linear generalization of the affine systems without drift. One can always find coordinates in which such systems have the form

$$
\frac{d x^{i}}{d t}=u^{i} \quad(1 \leq \ldots \leq n-1), \quad \frac{d x^{n}}{d t}=g(x, u),
$$

where $g$ is homogeneous of degree one in $u^{i}$.

II) Systems which are hyperplanes in each fiber, these are the affine control systems

$$
\frac{d x}{d t}=A(x)+B(x) u .
$$

The case $A(x)=0$ is the case of no drift, which is then also of type $\mathrm{I}$.

III) Systems whose Pick cubic form in each fiber are zero. In this case, the fibers are hyperquadrics and those of maximal rank then have a non-degenerate quadric in each fiber and hence a feedback invariant pseudo-Riemannian metric whose geodesics are known to be the time optimal trajectories [5].

Finally we observe that additional structures of control systems on manifolds can usefully be extended to this degree of generality. In particular in optimal control problems there is a cost function whose integral along paths is studied. This gives rise to a more complicated equivalence in which simple equivalence of Lagrangians (see [4]) needs to be added, as well as possibly modification of the integrand by divergences.

\section{References}

[1] W. Blaschke, Vorlesungen Über Differentialgeometrie II, Springer, Berlin, 1923.

[2] R. Brockett, Feedback Control of Linear and Non-linear Systems, Lecture Notes in Control and Information Science, vol. 39, Springer, New York, 1982.

[2a] R. Brockett and X. Dai, The dynamics of the ball and plate problem, preprint, 1993.

[3] R. Bryant, On notions of equivalence of variational problems with one independent variable, Contemporary Mathematics 68 (1987), 65-76.

[4] R. Gardner, The Method of Equivalence and Applications, SIAM-CBMS Regional Conf. Ser. in Appl. Math. 58, Philadelphia, 1989.

[5] R. Gardner, W. Shadwick and G. Wilkens, A geometric isomorphism with applications to closed loop controls, SIAM J. Control and Optim. 27 (1989), 1361-1368.

[6] R. Gardner and G. Wilkens, Classical geometries arising in feedback equivalence, in: Proceedings of the 32nd IEEE-CDC, San Antonio, Texas, 1993. 